\title{
BIBECHANA
}

A Multidisciplinary Journal of Science, Technology and Mathematics ISSN 2091-0762 (Print), 2382-5340 (0nline) Journal homepage: http://nepjol.info/index.php/BIBECHANA Publisher: Research Council of Science and Technology, Biratnagar, Nepal

\section{Physical properties of dust particles around white dwarf in far infrared sky at $12.8^{0}$ galactic latitude}

\author{
B. B. Sapkota ${ }^{1 *}$ B. Aryal' ${ }^{2}$, R. Weinberger ${ }^{3}$ \\ ${ }^{1}$ Mahendra Ratna Campus, Tahachal, Tribhuvan University, Kathmandu, Nepal. \\ ${ }^{2}$ Center Department of Physics, Tribhuvan University, Kirtipur, Nepal. \\ ${ }^{3}$ Institute of Astro-Particle Physics, Insbruck University, Austria. \\ *Email: bhanusapkota45@gmail.com
}

Article history: Received 15 October, 2017; Accepted 12 November, 2017

DOI: http://dx.doi.org/10.3126/bibechana.v15i0.18488

This work is licensed under the Creative Commons CC BY-NC License.

https://creativecommons.org/licenses/by-nc/4.0/

\section{Abstract}

We study the active region in the interstellar medium in which the process of cavity formation is expected. The interaction between wind and its surroundings in the interstellar medium (ISM) provides a laboratory to study the behavior of dust particles. The information obtained from the dust colour temperature and dust mass of WD2116+675 will be presented. It is found that the dust colour temperature and dust mass lie in the range $20 \mathrm{~K}$ to $22.3 \mathrm{~K}$ and $2.1 \times 10^{23} \mathrm{~kg}$ to $2.9 \times 10^{23} \mathrm{~kg}$ respectively. With the help of number density, flux density, dust colour temperature and dust mass maps, the variation of temperature and mass in different position of dust will be presented and discussed.

Keywords: White dwarfs; Flux Density; Cavity.

\section{Introduction}

White dwarf are hot $(\sim 10,000 \mathrm{~K})$ low luminosity stars composed mostly of carbon, oxygen and helium. Their luminosity is low because their surface is small. The electrons in the white dwarf are degenerate. The largest possible white dwarf is 1.4 of $\mathrm{M}_{\text {sun. }}$. This is called Chandrasekhar limit, and is the most mass that the electron degenerate core can support without collapsing under its own gravity. No white dwarf with masses less than $0.6 \mathrm{Msun}$ have been observed yet. The matter and radiation that exists in the space between the star system in galaxy is called Interstellar Medium (ISM). The ISM is the dust and gas between the stars. $99 \%$ of ISM is gas, and only $1 \%$ of the mass is dust. About $20 \%$ of the galaxies mass is ISM [1].

The interstellar dust consists of particles of silicates or carbon. The gas is mostly hydrogen gas, atomic and molecular gas. Dust emits in the infrared but blocks visible light. The reduction in amount of light or other radiation received from star as a result of absorption and scattering of the radiation by intervening dust grains in space is called extinction. The extinction decreases with increase of wavelength of the radiation and increases with the path length through the absorbing medium and 
with the density of the medium. Blue light is scattered and absorbed more than red in interstellar medium. This effect is called reddening of light. In other words, the extinction of blue light by dust is greater than red light [2].

In the present work we intend to study a new cavity- like structure around White dwarf.

\section{Region of Interest and Methods}

We have compiled a database of 1978 number of white dwarfs which are listed in the catalogue of Holberg et. al.[3]. Out of which we have studied WD2116+675 here. We have carried out a systematic search of IRAS maps available in the sky view virtual observatory (http://skyview.gsfc.nasa.gov). This sample white dwarf has the right ascension of $20^{\mathrm{h}} 17^{\mathrm{m}} 17^{\mathrm{s}}$ and declination of $+67^{0} 44^{\mathrm{m}} 44^{\mathrm{s}}$ in equatorial coordinate system. The following input parameters were used for the search:

(1) Coordinate: J2000, (2) Projection: Gnomonic (Tan), (3) Image size (pixel): 500×500 (4) Image size (degrees) : $0.5^{0} \times 0.5^{0}$ (5) Brightness Scaling: Histogram Equilization (HistEq) (6) Colour Table: Stern Special.

We have downloaded Flexible Image Transport System (FITS) v3 image of the selected region. We selected FITS format of $0.5^{0} \times 0.5^{0}$ at 60 and $100 \mu \mathrm{m}$ for the image processing. Using ALADIN v2.5 software the FITS image carries the information concerning the flux density, position, etc for each pixels.

Aladin v2.5 is an interactive sky atlas developed and maintained by the Center de Donne's astronomiques de Strasbourg (CDS) for the identification of astronomical sources through visual analysis of reference sky images. Aladin v2.5 allows the user to visualize digitized images of any part of the sky, to superimpose entries from the CDS astronomical catalogues and tables, and to interactively access related data and information from SIMBAD, NED or other archives of all known objects in the field.

In ordered to separate the region of minimum flux density region, contours are drawn at 60 and 100 $\mu \mathrm{m}$ respectively. Because we are interested to study temperature and mass profile of the region. We intend to study the cavity - like structure at $60 \mu \mathrm{m}$ and $100 \mu \mathrm{m}$.

Measured flux density is subtracted with the background values. Background flux is the flux emitted by other sources lying nearby the region of interest (not from the region of interest). The average value of the background flux is obtained by noting and summing up of the minimum flux densities around the region of interest and dividing the sum by total number of pixel with this minimum flux density. When this background flux is subtracted from the obtained flux density of each pixels in the region of interest, it is said to be background flux density.

\section{Dust color temperature estimation}

We use data base from the IRAS $60 \mu \mathrm{m}$ and $100 \mu \mathrm{m}$ flux densities is similar to that of Schnee et. al.[4]. By knowing the flux densities at $60 \mu \mathrm{m}$ and $100 \mu \mathrm{m}$, the temperature contribution due to dust color can be calculated. The dust temperature $T_{d}$ in each pixel of a FITS image can be obtained by assuming that the dust in a single beam is isothermal and that the observed ratio of $60 \mu \mathrm{m}$ to $100 \mu \mathrm{m}$ emission is due to black body radiation from dust grains at $T_{d}$, modified by a power law of spectral emissivity index. The flux density of emission at awavelength $\lambda_{i}$ is given by

$$
\left.F_{i}=\left[\frac{2 h c}{\lambda_{i}^{3}\left(e^{\frac{h c}{k T_{d}}}-1\right)}\right] N_{d} \alpha \lambda_{i}^{-\beta} \Omega_{i}\right)
$$


where, $N_{d}$ is the column density of dust grains, is a constant which relates the flux withthe optical depth of the dust, $\beta$ is the spectral emissivity index, and $\Omega_{i}$ is the solid angle subtended at $\lambda_{i}$ by the detector. Following [6], we use the equation

$$
\beta=\frac{1}{\delta+\omega T_{d}}
$$

To describe the observed inverse relationship between temperature and emissivity spectral index. Here, $\delta$ and $\omega$ are free parameters found that the temperature dependence of the emissivity index fits very well with the hyperbolic approximating function.

Considering temperature as an independent variable, the best fit gives $\delta=0.40 \pm 0.02$ and $\omega=0.0079$ $\pm 0.0005 K^{-1}$, with the $\chi^{2} /$ degree of freedom $=120 / 120$. With the assumptions that the dust emission is optically thin at $60 \mu \mathrm{m}$ and $100 \mu \mathrm{m}$ and that $\Omega_{\omega} \cong \Omega_{100}$ (true for IRAS image), we can write the ratio "R" of the flux densities at $60 \mu \mathrm{m}$ and $100 \mu \mathrm{m}$ as

$$
R=0.6^{-(3+\beta)} \frac{e^{\frac{144}{T_{d}}}-1}{e^{\frac{240}{T_{d}}}-1}
$$

The value of $\beta$ depends on dust grain properties as composition, size, and compactness. For reference, a pure blackbody would have $\beta=0$, the amorphous layer-lattice matter has $\beta \sim 1$, and the metals and crystalline dielectrics have $\beta \sim 2$. For a smaller value of $T_{d}, 1$ can be dropped from both numerator and denominator of equation and it takes the form

$$
R=0.6^{-(3+\beta)} \frac{e^{\frac{144}{T} d}}{e^{\frac{240}{T} d}}
$$

Taking natural logarithm on both sides of equation (4) we find the expression for the temperature as

$$
T_{d}=-96 \frac{1}{\ln \left\{R \times 0.6^{(3+\beta)}\right\}}
$$

where $\mathrm{R}$ is given by

$$
\mathrm{R}=\frac{\mathrm{F}(60 \mu \mathrm{m})}{\mathrm{F}(100 \mu \mathrm{m})}
$$

$\mathrm{F}(60 \mu \mathrm{m})$ and $\mathrm{F}(100 \mu \mathrm{m})$ are the flux densities at $60 \mu \mathrm{m}$ and $100 \mu \mathrm{m}$, respectively. In this way we can use equation (5) for the determination of the dust grain temperature [6].

\section{Mass Estimation}

Since the longer wavelength measurements give us more precise dust masses due to the characteristics of the Planck curve, the far infrared emission which is used for the derivation of the dust mass is measured from the $100 \mu \mathrm{m}$ IRAS images. The dust masses are estimated from the IR flux densities. In order to estimate the dust masses from the infrared flux densities at $100 \mu \mathrm{m}$, followingthe calculation of Young et. al.[7]. We need the background correction of flux and convert the relative flux into absolute flux. The background correction is done by subtracting the average flux emitted by the external sources other than the object of interest. The blackbody intensity can be calculated using the basic expression as given in equation (2). The resulting dust mass depends on the physical and chemical properties of the dust grains, the adopted dust temperature $T_{d}$ and the distance $D$ to the object.

$$
M_{d u s t}=\frac{4}{3} \frac{a \rho}{Q_{v}}\left[\frac{S_{v} D^{2}}{B(v, T)}\right]
$$

where $\mathrm{a}, \rho, \mathrm{Q}_{\mathrm{v}}$ and $\mathrm{s}_{\mathrm{v}}$ represent weighted grain size, grain density, grain emissivity and flux density of the region of interest, respectively. Here,

$$
\mathrm{S}_{v}=\mathrm{f} \times 5.288 \times 10^{-9} \frac{\mathrm{MJy}}{\mathrm{S}}
$$

The distance (D) to the cavity is $205 \mathrm{pc}$, known from Odenwald et .al.[5].

The Planck's function is given by,

$$
\mathrm{B}(v, \mathrm{~T})=\frac{2 \mathrm{~h} v^{3}}{\mathrm{c}^{2}}\left[\frac{1}{\mathrm{e}^{\mathrm{kT}}-1}\right]
$$


where h,c, $v$, and T represent Planck's constant, velocity, frequency of light, and average temperature of region respectively.

By using, $a=0.1 \mu \mathrm{m}[5], \rho=3000 \mathrm{kgm}^{-3}$, and $\mathrm{Q}_{v}=0.0010$ for $100 \mu \mathrm{m}$ and 0.0046 for $60 \mu \mathrm{m}$ respectively [6] ,the expression (7) takes the form :

$$
\mathrm{M}_{\text {dust }}=0.4\left[\frac{\mathrm{S}_{v} \mathrm{D}^{2}}{\mathrm{~B}(\mathrm{v}, \mathrm{T})}\right]
$$

We use the equation (9) for the calculation of the dust mass

It is clear from the expression (8) that the value of Planck function $B(\nu, T)$ for longer wavelength is higher than that of the shorter wavelength. Consequently, the range of $B(v, T)$ for fixed temperature $(\operatorname{say} \Delta \mathrm{T})$ goes narrower if wave-length of the images increases.

\section{Results and Discussion}
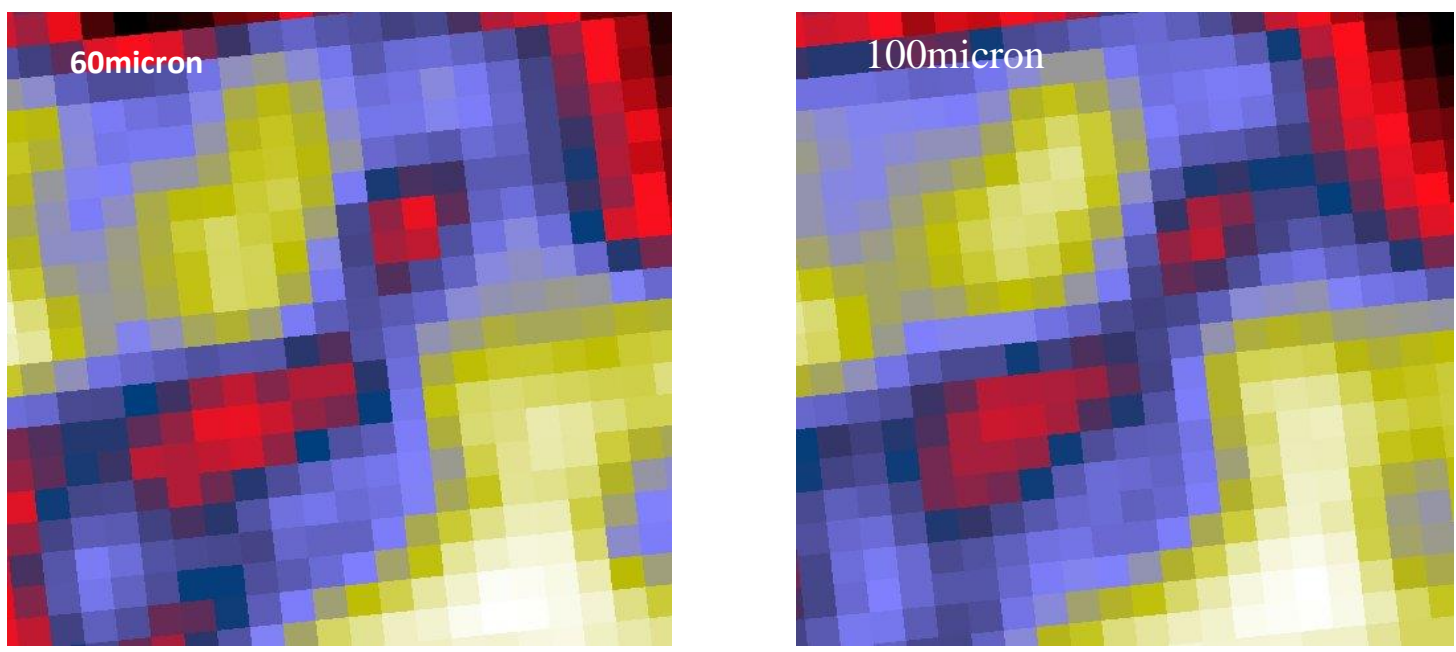

Fig. 1: $0.5^{0} \times 0.5^{0}$ image of the region centered at R.A. $(\mathrm{J} 2000)=21^{\mathrm{h}} 17^{\mathrm{m}} 17^{\mathrm{s}}$,Dec. $(\mathrm{J} 2000)=+67^{0} 44^{\prime} 44^{\prime \prime}$ at $60 \mu \mathrm{m}$ (left) and $100 \mu \mathrm{m}$ (right) of WD $2116+675$.
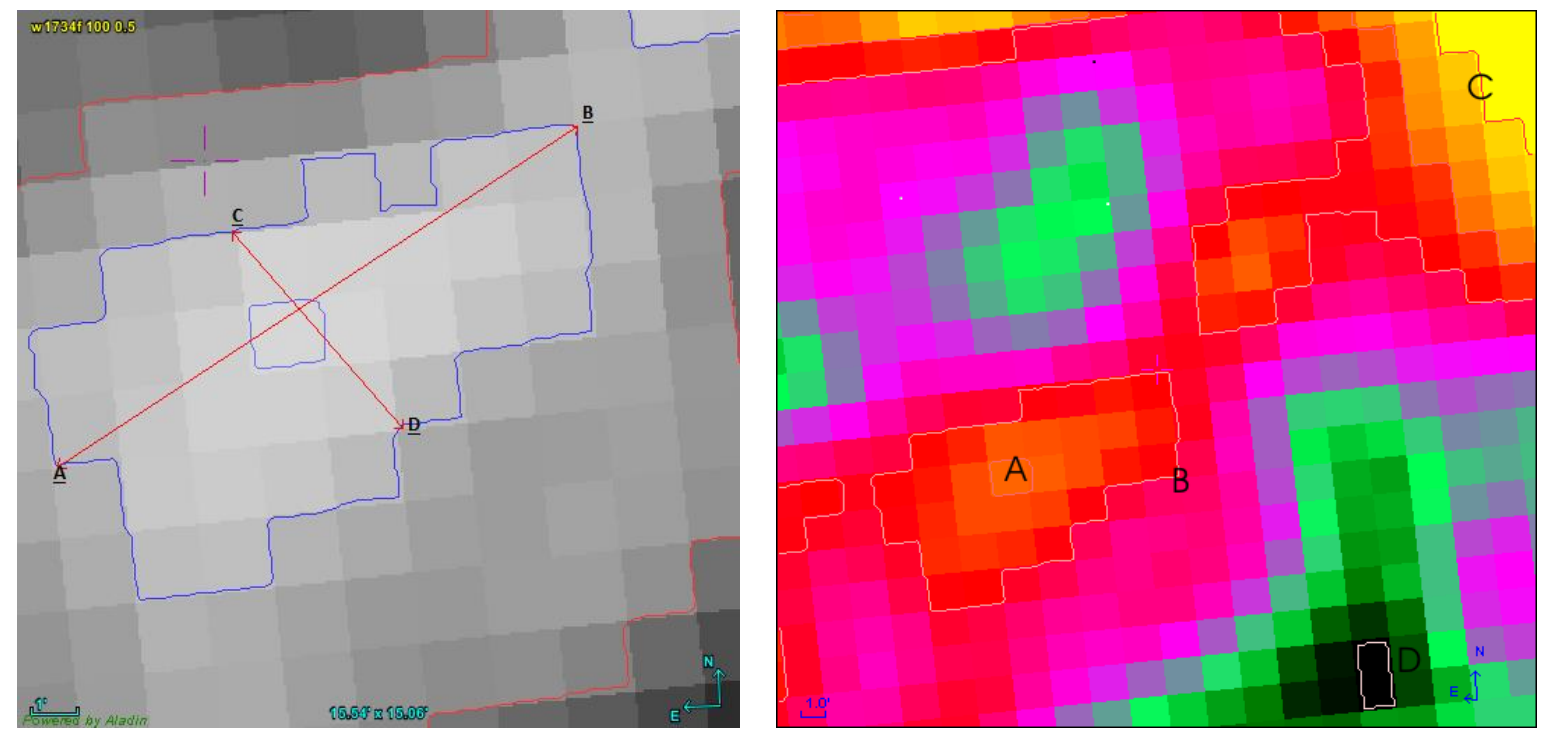

Fig 2

Fig.3 
Fig. 2: Cavity with contour and major diameter $(A B)$ and minor diameter $(C D)$. The size of image is $0.5^{0} \times 0.5^{0}$ at $100 \mu \mathrm{m}$ IRAS maps.

Fig. 3:Showing different flux density inside the different contour level outside whole region of WD2116+675 centered at R.A. $=21^{\mathrm{h}} 17^{\mathrm{m}} 17^{\mathrm{s}}$ and Dec. $=+67^{\circ} 44^{\prime} 44^{\prime \prime} .+$ sign represents position of white dwarf and A, B, C, and D represents four contour level drawn at 2, 40, 69, and 255 respectively.

Figure (1) represents 60 and $100 \mu \mathrm{m}$ IRAS image of the region of interest. The region of minimum and maximum flux is represented by black and white colors respectively. Diameters are drawn passing through minimum flux inside the cavity as shown in figure (2). Figure (3) shows four contour level A, B, C, and D that surround flux density 23.86,25.75, 25.02 and $30.57 \mathrm{MJySr}^{-1}$ respectively. Contour A encloses minimum flux density and B encloses maximum flux density inside the cavity. Similarly, contour level C , and D encloses minimum and maximum flux density including whole region of the region. The flux density obtained inside the contour level $\mathrm{C}$ helps to determine background count. Similarly, the flux density of other required region can be obtained with the help of Aladin software. By comparing Figures (1), (2) , and (3), we get same result.

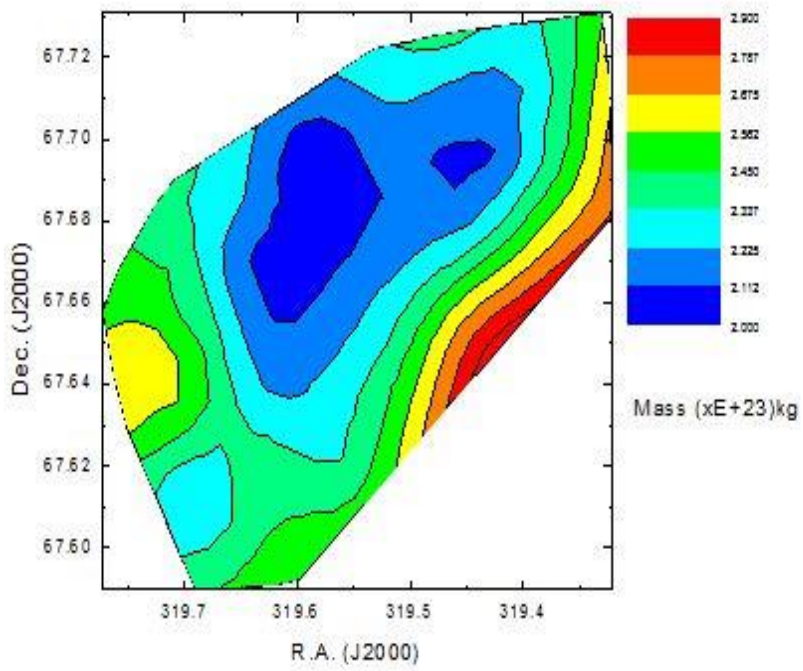

Fig. 4

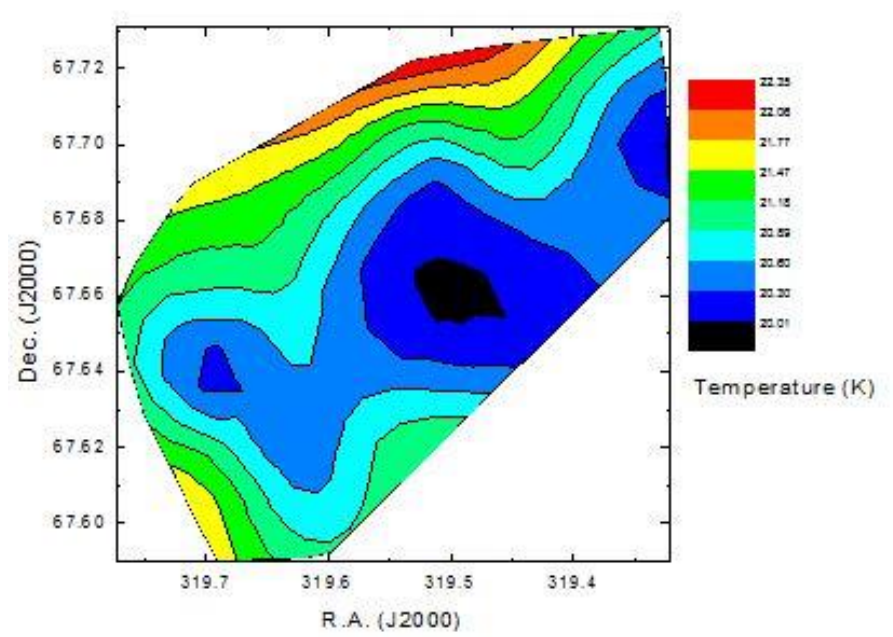

Fig. 5

Fig 4 : Contour map of mass distribution. The map is centered at R.A.(J2000) $=21^{\mathrm{h}} 17^{\mathrm{m}} 17^{\mathrm{s}}$, Dec. $(\mathrm{J} 2000)=+67^{\circ} 44^{\prime} 44^{\prime \prime}$.

Fig. 5: Dust colour temperature contour plot. The map is centered at R.A.(J2000) $=21^{\mathrm{h}} 17^{\mathrm{m}} 17^{\mathrm{s}}$, Dec. $(\mathrm{J} 2000)=+67^{0} 44^{\prime} 44^{\prime \prime}$.The contour levels are shown.

In figure (4), different colour counter represents the different range of mass. From above plot mass is higher at centre and decreases across the outward region. Figure (5) shows the different colour map of temperature. From above plot temperature is lower at centre and increases across the outward region. By comparing figure (4) and figure (5) we get those region at which mass is minimum, greater the value of temperature of dust. 


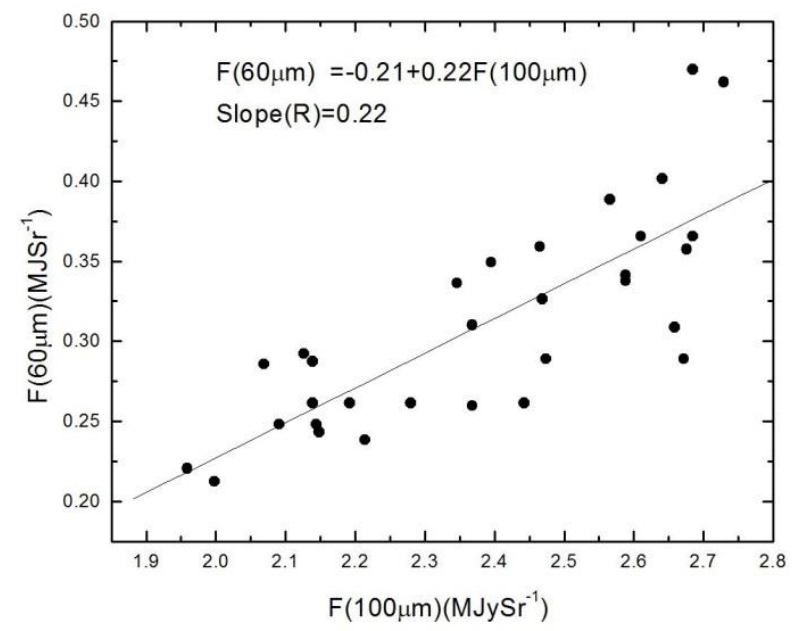

Fig. 6

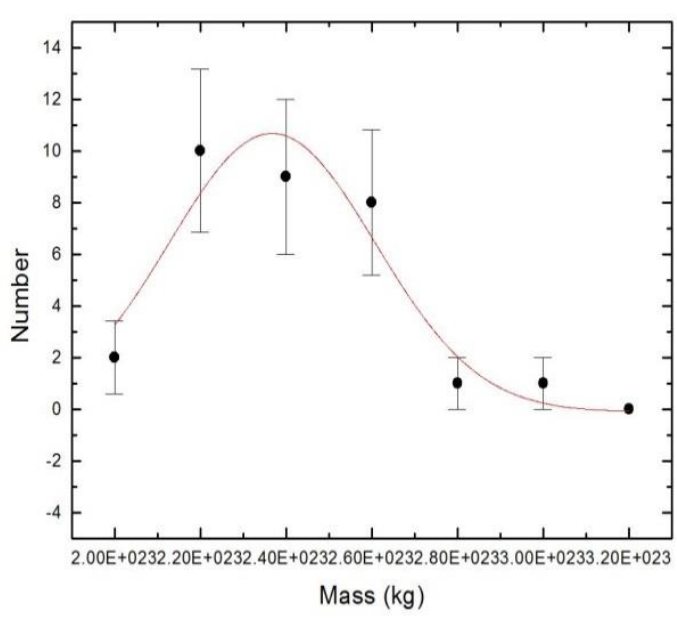

Fig. 7

Fig. 6: The flux density at $60 \mu \mathrm{m}$ versus $100 \mu \mathrm{m}$ plot. The best fit can be seen.

Fig. 7: Dust mass distribution. The Gaussian fit and the $\pm 1 \sigma$ statistical error bars can be seen. Here $\sigma=\sqrt{ }$.

Figure (6) is the plot of relative flux density of $60 \mu \mathrm{m}$ versus $100 \mu \mathrm{m}$. The solid line represents linear fit. The slope of the line has been used to find average dust color temperature of the region of interest. Figure(7) shows the Gaussian plot of mass distribution. The solid curve represents Gaussian fit of mass with error bar. The dust mass is very well fitted. The Gaussian parameter i.e. Gaussian centre is $2.37 \times 10^{23} \mathrm{~kg}$ and offset mass is $-0.11 \mathrm{~kg}$.

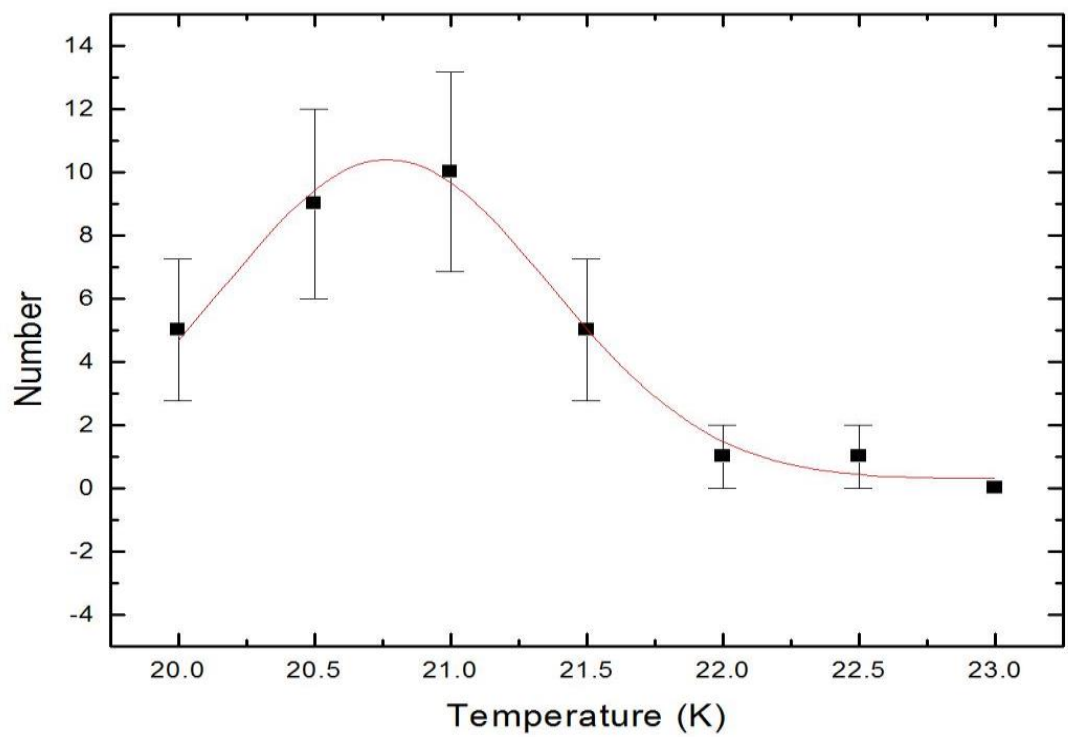

Fig. 8: Distribution of dust color temperature. The Gaussian fit and the $\pm 1 \sigma$ statistical error bars can be seen. Here $\sigma=\sqrt{ }$. 
Figure(8) shows the Gaussian plot of dust color temperature The solid curve represents Gaussian distribution. The dust color temperature is well fitted as Gaussian distribution. The offset temperature is $0.28 \mathrm{~K}$ and Gaussian centre is $20.77 \mathrm{~K}$. This suggest that the cavity is in thermal equilibrium and not effected by external cause. There is less deviation in temperature.

\section{Conclusions}

The maximum and minimum dust color temperature of structure WD2116+675 is found to be $22.34 \mathrm{~K}$ and $20.02 \mathrm{~K}$ respectively. Total mass of dust structure is found to be $7.42 \times 10^{24} \mathrm{~kg}$. The dust mass and dust colour temperature is found to be well fitted by Gaussian distribution. The size of cavity is $0.78 \mathrm{pc} \times 0.329 \mathrm{pc}$. The cavity is found to be formed due to presence of nearby white dwarf. The white dwarf evolves from the planetary nebula stage by ejecting its outer envelopes.

\section{Acknowledgements}

We thank editor and referee for the constructive comments and suggestion. One of the authors (BBS) thanks University Grants Commission of Nepal for providing research fund and acknowledge Central Department of Physics, Tribhuvan University , Nepal for various kinds of support during $\mathrm{Ph} . \mathrm{D}$. In addition, we acknowledge sky view virtual observatory ( http://skyview.gsfc.nasa.gov ) SIMBAD ( http://skyview.gsfc.nasa.gov ) for providing database and software.

\section{References}

[1] S. Palen, Schaum's Outlines Astronomy, MC Graw Hill, USA, 2004.

[2] H. Karttunen, P. Kroeoger, H. Oja, M. Poutanen, K.J. Donner, Fundamental Astronomy, Springer Berlin Heidelberg, Fifth Edition. USA, 2007.doi.org/10.1007/978-3-540-34144-4.

[3] J. B. Holberg, Terry D. Oswalt,and E. M. Sion., A determination of the local density of white dwarf stars Astrophysical Journal 571 (2002) 512-518. doi.org/10.1086/339842.

[4] S. L.Schnee, N. A. Ridge, N.A., Goodman, A.A, Jason, G. L., A COMPLETE look at the use of IRAS emission maps to estimate extinction and dust temperature, Astrophysical Journal 634 (2005) 442- 450. doi.org/10.1086/491729.

[5] S. F. Odenwald and L. J. Richard, Hydrodynamical Process in the Draco, Astrophysical Journal 318 (1987) 702-711.

[6] X. Dupac, J.P. Bernard, N Boudet, M. Giard, J.M. Lamarre, C Meny, F. Pajot, I. Ristorcell., G. Serra, B. Stepnik, J.P. Torre, Inverse temperature dependence of the dust submillimeter spectral index Astronomy \& Astrophysics 404 (2000) L11-L15. doi.org/10.1051/0004-6361:20030575.

[7] K. Young., T.G. Philip., \& G.R. Knapp, Circumstellar shells resolved in IRAS survey data. II Analysis, Astrophysical Journal, 409, (1993) 725-738. doi.org/10.1086/172702 